Z. klin. Chem. u. klin. Biochem.

7. Jg., S. $379-383$, Juli 1969

\title{
Zur Problematik des Harnsammelns und -konservierens bei der gaschromatographischen Steroidhormonbestimmung
}

\author{
Von H. Gleispach \\ Aus der Universitäts-Kinderklinile Innsbruck (Vorstand: Prof. Dr. H. Berger)
}

(Eingegangen am 22. März 1969)

\begin{abstract}
Die angeführten Untersuchungen zeigen deutlich, daß eine r $^{\mathrm{i}}$ chtige Konservierung des Harnes sowohl beim Lagern als auch beim Sammeln unbedingt erforderlich ist. Eine Rekonstruktion der eigentlichen Werte nach Bestimmung der Steroidverteilung in einem unsachgemäß gesammelten oder gelagerten Harn ist nicht möglich. Somit ist eine Hormonanalyse nur sinnvoll, wenn die Konservierung während der Sammelperiode sowie der anschließenden Aufbewahrung einwandfrei durchgeführt war. Nach unseren bis jetzt durchgeführten Untersuchungen erhält man nach folgender Vorschrift die besten Ergebnisse:

In das Sammelgefäß gibt man 3 Tropfen Chloroform und etwa $1 / 10$ Vol. der zu erwartenden Harnmenge Natriumacetatpuffer pH 4,0. Nach dem Sammeln der 24-Stunden-Harnmenge wird das Chloroform mit Stickstoff (nie Preßluft verwenden) abgeblasen. Unterläßt man das Abblasen des Chloroforms, so erleidet man Verluste, bedingt durch die im Chloroform gelösten Steroide. Nach Abblasen des Chloroforms mißt man das Gesamtvolumen. Anschließend inkubiert man einen aliquoten Teil des Harnes für die gaschromatographische Bestimmung. Will man einen Teil des Harnes für spätere Untersuchungen aufbewahren, so fülle man die für je eine Untersuchung benötigte Menge Harn in je eine Flasche, füge einige Tropfen Chloroform hinzu und friere ein. So kann man bei einer späteren Untersuchung die benötigte Menge auftauen und vermeidet Verluste, die durch mehrmaliges Auftauen und Einfrieren entstehen.
\end{abstract}

\section{The collection and preservation of urine for the gas chromatographic determination of steroid bormones}

The results of the reported experiments show plainly that the correct preservation of urine both in storage and during collection is absolutely essential. Correction of the actual values after the determination of steroid distribution in an improperly collected or stored urine is not possible. A hormone analysis is meaningful only if the preservation during the collection period and subsequent storage is satisfactory. According to our studies so far, the best results are obtained as follows:

Three drops of chloroform and a volume of sodium acetate buffer $\mathrm{pH} 4.0$ representing about one-tenth of the expected urine volume are added to the collection vessel. After urine collection for $24 \mathrm{hr}$, the chloroform is blown off with nitrogen; compressed air must not be used. The steroids dissolved in the chloroform are lost if the chloroform is not removed by evaporation. The total urine volume is then measured, and an aliquot is incubated for the gas chromatographic determination. If part of the urine must be stored for later investigation, the amount of urine required for each separate determination is placed in a bottle with a few drops of chloroform and frozen. Thus the losses incurred by repeated freezing and thawing are avoided, if only the required amount need be thawed for each later investigation.

Die Veränderungen, denen die Steroidhormone bei Lagerung im kristallinen Zustand wie auch in Alkohol und Harn gelöst unterworfen sind, werden untersucht und eine Vorschrift zur Konservierung der Harnsteroide wird angegeben. Die Zersetzung der kristallinen Steroide kann durch trockene Lagerung bei tiefen Temperaturen weitgehend ausgeschaltet werden. Bei den im Harn gelösten Steroiden sind sowohl durch Einfrieren und Wiederauftauen, durch Lagerung im gefrorenen Zustand und vor allem durch Stehen bei Zimmertemperatur starke Veränderungen beobachtet worden. Daraus ergibt sich die Forderung nach einer wirksamen Konservierung der Harnsteroide bereits während der Sammelperiode, soll eine gaschromatographische Bestimmung wirklich die tatsächliche Verteilung der Steroide und nicht Artefakte erfassen.

Die gaschromatographische Bestimmung der Steroidhormone setzt sich immer mehr, auch im klinischen Routinebetrieb, durch. Die allgemein gebräuchliche Methode ist die Fraktionierung der im Harn vorkommenden Androgene und Pregnane. Wesentlich für die Genauigkeit der Analyse ist die Unversehrtheit des Harnes. Somit stellt sich die Frage, wie das Sammeln des Harnes durchgeführt werden muß, damit die bestimmten Hormone auch wirklich mit den ausgeschiedenen iden- tisch sind und nicht Artefakte das Hormonmuster beträchtlich verändern. Auch ist es vielfach üblich, die Harnproben nicht sofort aufzuarbeiten - sei es, weil es im Routinebetrieb einfacher ist, nicht täglich die anfallenden Harne sofort $z u$ inkubieren, sondern eine gewisse Anzahl Harne zu sammeln und dann gemeinsam $z u$ verarbeiten - sei es, weil man z. B. vor der gaschromatographischen Bestimmung sich mit einer der Sammelbestimmungsmethoden für die 17-Ketosteroide oder 17-Hydroxycorticoide einen Überblick verschaffen will, bei welchen Harnen sich eine gaschromatographische Analyse lohnt. Will man aber einen Harn längere Zeit aufbewahren, so muß man sich über die Veränderungen, die das Hormonmuster unter den verschiedenen Aufbewahrungsmöglichkeiten erleidet, im klaren sein. In der Literatur sind die Veränderungen, die das Hormonmuster einerseits bei unsachgemäßem Harnsammeln, andererseits bei längerer Aufbewahrung erleidet, nicht eingehend behandelt. In H. H. Wotrz und ST. J. CLARK "Gaschromatography in the analysis of steroid hormones" (1) wird zwar angegeben, welches Aliquot eines 24-Stunden-Harnes für diese oder jene Bestimmung $\mathrm{zu}$ verwenden ist, nicht aber wie der Harn gesammelt oder aufgehoben werden soll. In H. J. HüBENER und W. H. StaIB „Biochemie der Nebennierenrindenhor- 
mone“ (2) heißt es: „Den besten Überblick über die Ausscbeidung von Steroidmctaboliten erbält man durch ibre Bestinmmung in der 24-Stunden-Harnmenge. Kürzere Sammelzeiten liefern unbefriedigende Ergebnisse, da die Ausscheidung einiger Steroide typischen Tagesscbusankungen unterworfen ist. Oftmals sind auf mangelhaftes Urinsammeln größere Bestimmungsfebler zuriickzufiibren als auf methodiscbe Unz,tlänglichkeit. Desbalb sollte man die Vollständigkeit einer 24Stunden-Harnmenge durch Bestimmung der Kreatininausscbeidung iiberpriifen. Eine 24-Stunden-Sammelperiode beginnt nach dem morgendlichen Harnlassen und endet nach dem morgendlicben Harnlassen des folgenden Tages.

Die Harnproben können längere Zeit im gefrorenen Zustand konserviert werden, wäbrend man sie bei $+5^{\circ}$. nur etwa längstens 25-30 Tage obne Veränderung im Steroidgebalt aufbenvabren kann.

Auch die Zugabe von Toluol, Chloroform oder Phenol scbützt die Steroide einige Zeit vor bakterieller Umuandlung. Vor der Aufarbeitung taut man die Harne vorsicbtig auf oder verdampft die zugegebenen 'Lösungsmittel und stellt das Originalvolumen durch Zulgabe von Wasser wieder her. Am besten ist es aber, die Proben sofort zu verarbeiten."

Eine sehr umfangreiche Aufstellung der bakteriellen Umwandlung der Steroide finden wir in der Monographie: „Metabolism of Steroid Hormones“ (3). Aber auch hier finden wir keine detaillierte Aufstellung der Umwandlungen, die sich bei üblicher Aufbewahrung im Harn vollziehen und die bei der Routinebestimmung zu berücksichtigen sind; oder Angaben darüber, wie man die Artefaktbildung vermeiden kann.

$\mathrm{Zu}$ Anfang unserer Untersuchungen der Harnsteroide hielten wir uns an die von HübENER und Staib (2) angegebene Vorschrift der Zugabe von Konservierungsmitteln bzw. an das Einfrieren der Harne. Sehr bald jedoch bemerkten wir Veränderungen in dem von uns verwendeten Testgemisch. Wir begannen darauf mit systematischen Untersuchungen betreffs Konservierung der Steroide in kristallinem Zustand und in alkoholischer Lösung sowie der im Harn gelösten Steroide.

\section{A rbeitsbedingungen (4)}

Ein aliquoter Teil des 24-Stunden-Harnes (die Menge variiert je nach der Aufgabenstellung) wird mit Essigsäure bzw. Natronlauge auf $\mathrm{pH} 6,2$ gebracht, dann fügen wir $20 \mathrm{~m} l$ Natriumacetat (1 $\mathrm{ar}$, mit Essigsäure auf $\mathrm{pH}$ 6,2 gebracht) einige Tropfen Chloroform (zur Aktivierung der $\beta$-Glucuronidase und als Konservierungsmittel) und 10000 Einheiten $\beta$-Glucuronidase hinzu (diese Mengenangaben beziehen sich auf je $100 \mathrm{~m} / \mathrm{Harn}$ ). Anschließend wird der Harn während 24 Stdn. bei $38^{\circ}$ inkubiert. Nach der Inkubation wird der Harn abgekühlt und die freien Steroide werden dreimal mit Äther extrahiert. Die noch im Harn verbliebenen sulfatisch gebundenen Steroide werden anschließend gespalten. Der Harn wird mit Schwefelsäure auf $\mathrm{pH} 0,8-1$ gebracht und in einer Extraktionsapparatur während 72 Stdn. mit Äther extrahiert. Die beiden Ätherextrakte werden je nach den gestellten Anforderungen vereinigt oder getrennt aufgearbeitet. Der Arbeitsgang bleibt in beiden Fällen der gleiche. Zuerst wird dreimal mit 2N Natriumcarbonat, dann mit Wasser neutral gewaschen. Der Äther wird abgedampft, Spuren Feuchtigkeit durch Zugabe und Abdampfen von Methanol im Vakuum aus dem Rückstand entfernt. Der Rückstand wird in Benzol aufgenommen und an Aluminiumoxid Woslm neutral einer säulenchromato- graphischen Vorreinigung und Trennung unterzogen. Wir erhalten zwei Fraktionen, beiden fügen wir je $100 \mu \mathrm{g}$ Androstendion als Standard zu, dann wird das Lösungsmittel abgedampft, der Rückstand mit wenig Äther in ein Spitzkölbchen überführt und nach Abblasen des Äthers entweder mit Hexamethyldisilazan und Trimethylchlorsilan (5) oder mit Bistrimethylsilylacetamid $(6,7)$ silyliert. Die Testsubstanzen werden nach Abblasen des Alkohols in gleicher Weise stabilisiert. Nach Abblasen des Silyliermittels im Stickstoffstrom werden die Steroide in $0,2 \mathrm{ml}$ Hexan gelöst und $0,1-0,5 \mu l$ dem Gaschromatographen injiziert. Die gaschromatographischen Bedingungen sind: Fraktometer F 20 Perkin Elmer, $2 \mathrm{~m}$ Glassäule mit 3\% XE 60 auf Gaschrom P, Säulenofen auf $220^{\circ}$, FID und Einspritzblock auf $260^{\circ}$, Trägergasstrom $\left(\mathrm{N}_{2}\right)$ $50 \mathrm{~m} / /$ Min., Abschwächung 1/16.

\section{Ergebnisse}

\section{Steroide in kristallinem Zustand bqu. alkobolischer Lösung}

Die ersten Veränderungen im Verlauf der Lagerung bemerkten wir bei Pregnantriol und Pregnantriolon. Bei beiden Substanzen stellten wir nach längerer Lagerung eine Abnahme der Peakhöhe im Gaschromatogramm im Vergleich zu den anderen Steroiden fest. Zur gleichen Zeit wie das Testgemisch hatten wir auch alkoholische Lösungen der Reinsubstanzen Androsteron, Ätiocholanolon, Dehydroepiandrosteron, 11-Ketoandrosteron, 11-Ketoätiocholanolon, 11- $\beta$-Hydroxyandrosteron, 11- $\beta$-Hydroxyätiocholanolon, Testosteron, Androstendion, Androstendiolon, Allo-Pregnandiol, Pregnandiol, Pregnantriol, Pregnantriolon, Aldosteron und Corticosteron hergestellt. Diese Lösungen waren im Kühlschrank bei $+5^{\circ}$ gelagert. Jede der verwendeten Substanzen war sofort nach der Herstellung der Lösung auf ihre Reinheit überprüft worden. Zu diesem Zweck wurde $1 \mathrm{~m} l$ der jeweiligen Lösung zur Trockene eingedampft, silyliert und nach Abblasen des Silylierreagens in Hexan gelöst dem Gaschromatographen injiziert. Bei den von uns verwendeten Steroiden (mit Ausnahme von Aldosteron und Corticosteron, welche besonders präpariert werden müssen) (8), wurde unter diesen Bedingungen nur ein Peak beobachtet. Dann wurde einer zweiten Probe Androstendion als Standard zugegeben und das Flächenverhältnis von $100 \mu \mathrm{g}$ Substanz zu $100 \mu \mathrm{g}$ Standard berechnet und eine Eichkurve erstellt, die zwar für die einzelnen Steroide verschiedene Steigung, aber stets Geradlinigkeit aufweist.

Nachdem nun im Testgemisch Veränderungen der Peakhöhe festgestellt wurden, wiẹderholten wir diese Versuche mit den am stärksten davon betroffenen Substanzen Pregnantriol und Pregnantriolon. In beiden Fällen mußten wir feststellen, daß außer den substanzspezifischen Peaks nun noch ein je nach Dauer der Lagerung größerer oder kleinerer vor allem aber sehr breiter Peak zu finden war. Auch die Flächengröße des dem Pregnantriol oder Pregnantriolon entsprechenden Peaks war dementsprechend kleiner als ursprünglich. Das Testgemisch enthielt $107 \mu \mathrm{g}$ Pregnantriol und $79 \mu \mathrm{g}$ Pregnantriolon pro $\mathrm{m} l$ Alkohol. Nach 6monatiger Lagerung ergab die Berechnung "nach der ursprünglich erstellten Eichkurve nur mehr eine Menge von $78 \mu \mathrm{g}$ Pregnantriol und $52 \mu \mathrm{g}$ Pregnantriolon. Noch bedeutend 
Abb. 1

Gaschromatogramm von Harn-Steroiden (vgl. Arbeitsbedingungen)

Die Bezeichnung der Steroide ist:

1 Androsteron

2 Ätiocholanolon

3 Dehydroepiandrosteron

4 Cholesterin (wird bei dieser Bestimmungsmethode nicht quantitativ erfaßt)

5 Testosteron

6 Androstendion als Standard
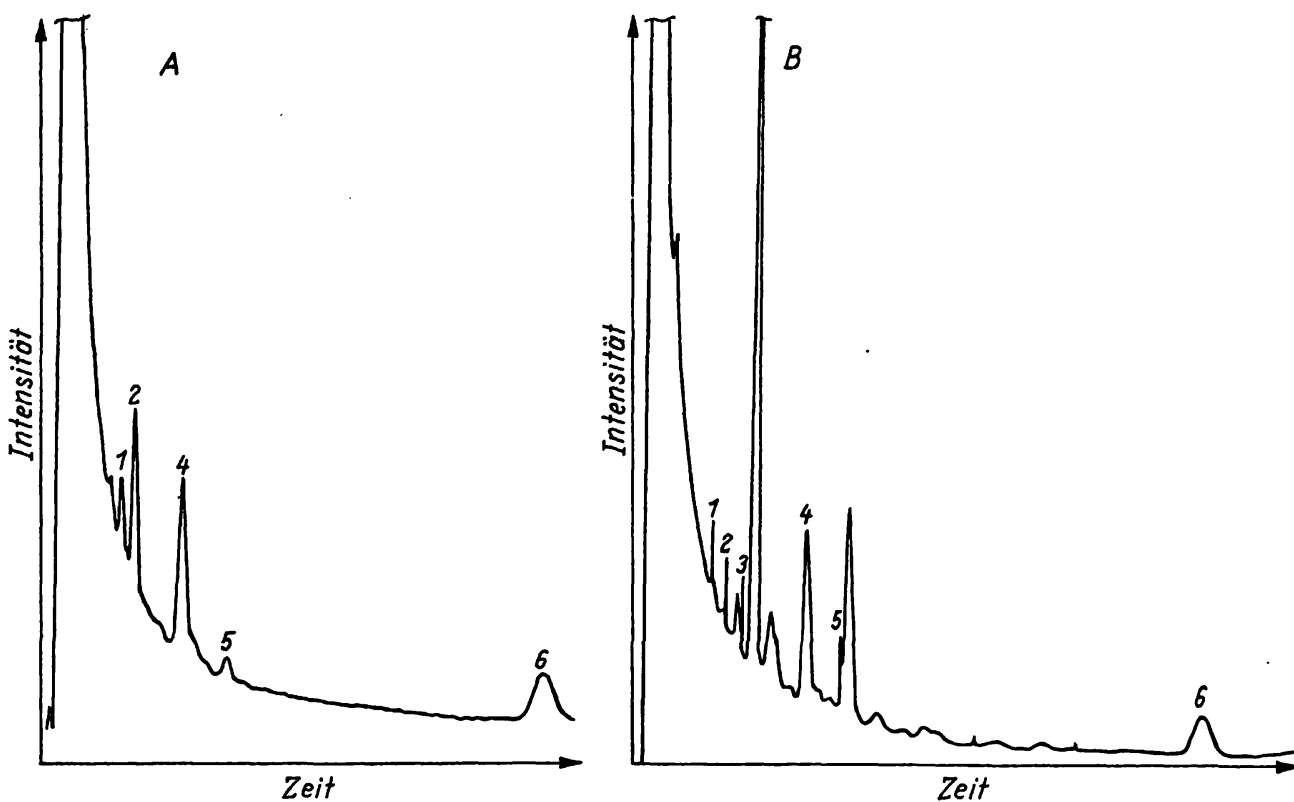

Die Unterschiede zwischen beiden Chromatogrammen (A und B) in den Retentionszeiten ergeben sich aus dem verschiedenen Alter des Säulenfüllmaterials. Die Retentionszeiten der Steroide wurden an Hand eines unmittelbar nachher injizierten Testgemisches in beiden Fällen bestimmt.

Chromatogramm A stellt die erste Fraktion nach Säulenchromatographie dar. Der Harn wurde unmittelbar nach Beendigung des Sammelns mit $\beta$-Glucuronidase inkubiert.

Chromatogramm B stellt dieselbe Fraktion des gleichen Harnes dar. Der Harn war jedoch 6 Monate bei - 20 eingefroren. Die Stellen der einzelnen oben angeführten Steroide wurden in das Chromatogramm eingezeichnet, um den Unterschied zu Chromatogramm A deutlicher zu gestalten.

Die vorhandenen Peaks entstammen offensichtlich Artefakten der in der zweiten Fraktion nun vermindert vorkommenden Steroide

schlechter waren die Ausbeuten bei Aldosteron und Corticosteron nach Lagerung einer alkoholischen Lösung. Bei Aldosteron fanden wir nach 6 Monaten nicht einmal 20\% der ursprünglichen Menge wieder. Bei den Androgenen waren die Verluste weniger drastisch. Pregnantriol und Pregnantriolon wie auch die übrigen Steroide hatten wir zu einem Teil in kristalliner Form im Kühlschrank bei $+5^{\circ}$ gelagert. Sofern die Packungen vor der Lagerung geöffnet worden waren, zeigten sich auch bei kristallinem Pregnantriol und Pregnantriolon Zersetzungsprodukte bzw. eine verminderte Ausbeute im Vergleich zu den frischen Packungen. Das in ungeöffneten Ampullen aufbewahrte Pregnantriol und Pregnantriolon zeigte keinerlei Veränderung ebenso wie das, welches nach der Öffnung, mit Silicagel verpackt bei $-20^{\circ}$ gelagert war.

Zusammenfassend kann man demnach sagen, daß die Steroide in alkoholischer Lösung (Äthanol p. A.) bei $+5^{\circ}$ im Kühlschrank nur eine begrenzte Zeit ohne merkliche Zersetzung aufbewahrt werden können. Dabei muß man allerdings feststellen, daß sich die Zersetzung von Steroid zu Steroid verschieden stark bemerkbar macht. Will man Steroide über einen längeren Zeitraum unzersetzt konservieren, so muß man sie in einer luftund feuchtigkeitsdichten Verpackung bei möglichst tiefer Temperatur aufbewahren.

\section{Steroide im Harn}

Auf Grund dieser Feststellung begannen wir daran zu $z w e i f e l n, \mathrm{da} \beta$ man einen Harn bei $-20^{\circ}$ beliebig lange aufbewahren könne, ohne eine Zersetzung befürchten zu müssen.
Deshalb wollten wir abklären:

1. Welche Veränderungen im Steroidmuster ergeben sich, wenn ein Harn 24 Stunden unter verschiedenen Bedingungen steht?

2. Welche Veränderungen ergeben sich lediglich durch das Einfrieren eines Harnes, und durch das Auftauen bei verschiedenen Temperaturen sowie, welches sind die günstigsten Bedingungen, unter denen man einen Harn einfrieren und auftauen kann?

3. Wie verändert sich ein bei $-20^{\circ}$ durch längere Zeit hindurch aufbewahrter Harn?

4. Kann man angeben, in welchem Verhältnis sich die einzelnen Steroide unter bestimmten Einflüssen verändern und inwieweit kann man einen älteren Harn noch verwenden?

Ad. 1. Ein Harn wurde in 8 Portionen geteilt. Je 2 Portionen wurden für eine Untersuchung verwendet. Die angegebenen Werte sind der Mittelwert der beiden Parallelbestimmungen.

a) Wurde sofort inkubiert,

b) einen Tag bei Zimmertemperatur stehengelassen und inkubiert,

c) wurde gleich b behandelt, jedoch wurden einige Kristalle Thymol zugegeben,

d) wie b behandelt, jedoch mit Essigsäure vorher auf $\mathrm{pH} 4,0$ gebracht und $20 \mathrm{ml} 1 \mathrm{M}$ Acetatpuffer $(\mathrm{pH} 4)$ je $100 \mathrm{ml}$ Harn sowie einige Tropfen Chloroform zugefügt.

Gaschrumatographisch bestimmt wurden in allen Fällen die freien und als Glucuronid gebundenen Steroide, die 
Tab. 1

Zersetzung der Harnsteroide durch Stehen des Harnes bei Zimmertemperatur während eines Tages, aufgezeigt an zwei Beispielen

Die angeführten Werte wurden wie folgt erhalten:

a) Sofortige Inkubation mit $\beta$-Glucuronidase und Extraktion der Steroide mit Äther. Die an Sulfat gebundenen Steroide wurden dann durch Ansäurern mit Schwefelsäure auf pH 0,8 und kontinuierliche Extraktion mit Äther gewonnen.

b) Der Harn wurde ohne Konservierungsmittel einen Tag bei Zimmertemperatur stehengelassen und dann mit $\beta$-Glucuronidase inkubiert.

c) Der Harn wurde gleich b behandelt, jedoch wurden einige Thymolkristalle zugegeben.

d) Der Harn wurde gleich b behandeit, er wurde jedoch vor dem sowie $1 \mathrm{ml}$ Chloroform je $100 \mathrm{ml}$ Harn zugegeben.

a) Freie und an Glucuronsäure gebundene Steroide wurden getrennt von den an Sulfat gebundenen Steroiden bestimmt

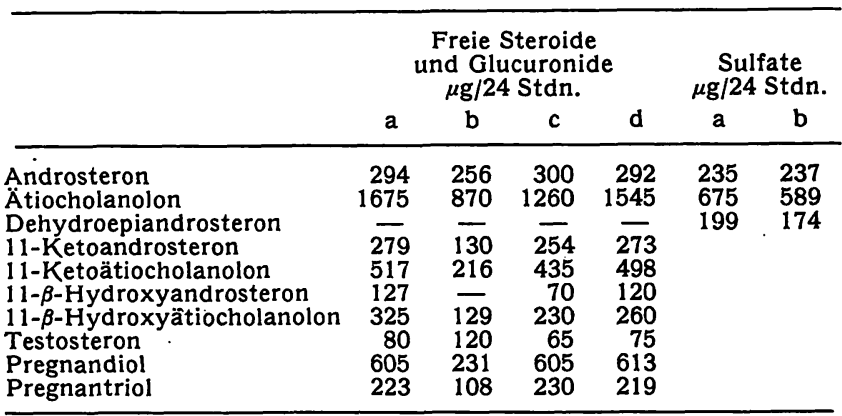

b) Es wurde jeweils die Summe der freien, der an Glucuronsäure sowie der an Sulfat gebundenen Steroide bestimmt

\begin{tabular}{|c|c|c|c|c|}
\hline & \multicolumn{4}{|c|}{$\begin{array}{l}\text { Gesamtsteroide } \\
\mu \mathrm{g} / 24 \text { Stdn. }\end{array}$} \\
\hline & $\mathbf{a}$ & b & c & d \\
\hline $\begin{array}{l}\text { Androsteron } \\
\text { Ätiocholanolon } \\
\text { Dehydroepiandrosteron } \\
\text { 11-Ketoandrosteron } \\
11-\text { Ketoätiocholanolon } \\
11-\beta \text {-Hydroxyandrosteron } \\
11-\beta \text {-Hydroxyätiocholanolon } \\
\text { Testosteron } \\
\text { Pregnandiol } \\
\text { Pregnantriol }\end{array}$ & $\begin{array}{r}678 \\
720 \\
41 \\
176 \\
286 \\
176 \\
125 \\
34 \\
525 \\
594\end{array}$ & $\begin{array}{r}653 \\
552 \\
35 \\
80 \\
155 \\
104 \\
70 \\
32 \\
411 \\
378\end{array}$ & $\begin{array}{r}675 \\
685 \\
43 \\
154 \\
217 \\
133 \\
108 \\
30 \\
507 \\
513\end{array}$ & $\begin{array}{r}680 \\
716 \\
40 \\
177 \\
278 \\
180 \\
121 \\
32 \\
531 \\
604\end{array}$ \\
\hline
\end{tabular}

Sulfate wurden nur bei a und $b$, nicht aber bei den übrigen Proben untersucht. Die Ergebnisse, aufgezeigt in Tabelle 1 an zwei der untersuchten Harne, demonstrieren eindringlich, daß ein Aufbewahren eines Harnes bei Zimmertemperatur ohne Zusatz eines Konservierungsmittels so gut wie unbrauchbare Ergebnisse liefert. Das bedeutet aber, daß sich auch das Steroidmuster während der Zeit des Harnsammelns ohne Zusatz eines Konservierungsmittels zumindest stark verzerrt, wenn nicht total verändert.

Bei Betrachtung der Tabelle zeigt sich aber auch, daß die Veränderungen, die die Hormone durch Zersetzung erleiden, nicht für sämtliche Steroide gleich bleiben. Sind die Veränderungen, die der Androsteronwert erleidet noch zu tolerieren, so verringert sich die Menge Ätiocholanolon innerhalb eines Tages fast auf die Hälfte, und 11- $\beta$-Hydroxyandrosteron verschwindet unter den Bedingungen des Versuchs $b$ nahezu vollständig. Im Gegensatz dazu steht Testosteron, welches offenisichtlich mikrobiell vermehrt wurde, so daß es bei Zugabe eines Konservierungsmittels geringe Verluste beim Stehen erleidet, während nach Stehen ohne Konservierungsmittel eine Zunahme der Testosteronmenge um die Hälfte des ursprünglichen Wertes erfolgt ist. Die als Sulfate ausgeschiedenen Steroide erleiden in den von uns untersuchten Fällen eine weit geringere Zersetzung als die frei oder als Glucuronid ausgeschiedenen Hormone.

Ad 2: Nachdem ein Aufbewahren des Harnes bei Zimmertemperatur während eines Tages untragbare Veränderungen im Hormonmuster mit sich bringt, gewinnt die Konservierung durch Einfrieren erhöhtes Interesse. Hierbei war erstens zu klären, ob sich der Harn beim Einfrieren und Auftauen verändert oder ob dadurch keine Verluste oder Veränderungen zu befürchten sind. Das zweite Problem, das es abzuklären gilt, ist, wie lange man einen Harn bei $-20^{\circ}$ ohne Veränderung des Hormonmusters konservieren kann. Tabelle 2 zeigt die Ergebnisse, die bei zwei der untersuchten Harne erhalten wurden.

Eine Portion eines Harnes wurde sofort nach Beendigung der Sammelperiode mit $\beta$-Glucuronidase inkubiert und aufgearbeitet. Weitere Portionen wurden eingefroren. Eine Portion wurde im fließenden heißen Wasser rasch aufgetaut und wieder eingefroren. Dies wurde dreimal wiederholt, dann wurde der Harn präpariert. Die nun gefundenen Werte betrugen weit weniger als $1 / 10$ der ursprünglich vorhandenen Mengen.

Mit den restlichen eingefrorenen Harnen stellten wir Versuche über die beste Möglichkeit des Auftauens an. Eine Portion wurde mit heißem fließendem Wasser aufgetaut, eine weitere im $40^{\circ}$ warmen Wasser und eine dritte durch Stehen bei Zimmertemperatur. Die besten

Tab. 2

Übersicht über die Zersetzung der frei und als Glucuronid ausgeschiedenen Steroide je nach Art des Auftauens

a) Sofortige Inkubation mit $\beta$-Glucuronidase

b) Inkubation erst nach mehrmaligem Einfrieren und Auftauen

c) Inkubation nach einmaligem Einfrieren und raschem Auftauen in siedendem Wasser

d) Inkubation nach einmaligem Einfrieren von $100 \mathrm{ml}$ Harn und Auftauen in $40^{\circ}$ warmen Wasser

e) Inkubation nach einmaligem Einfrieren. Vor dem Einfrieren wurde der Harn auf pH 4,0 gebracht und $20 \mathrm{ml}$ Natriumacetatpuffer sowie $1 \mathrm{ml}$ Chloroform je $100 \mathrm{ml}$ Harn zugegeben. Aufgetaut wurde bei $40^{\circ}$.

a) Harn einer 33jährigen Frau mit starkem Hirsutismus und Dysmenorrhoe (Testosteron wurde nicht bestimmt)

\begin{tabular}{lrrrrrr}
\hline & \multicolumn{5}{c}{ Freie Steroide und Glucuronide } \\
& \multicolumn{1}{c}{ a } & \multicolumn{1}{c}{ b } & c & \multicolumn{1}{c}{ d } & e \\
\hline Androsteron & 1010 & 530 & 825 & 950 & 1020 \\
Ätiocholanolon & 2010 & 231 & 1570 & 1780 & 1870 \\
Dehydroepiandrosteron & 216 & 83 & 160 & 190 & 187 \\
11-Ketoandrosteron & 1600 & 170 & 1240 & 1460 & 1600 \\
11-Ketoätiocholanolon & 2490 & 165 & 1800 & 1760 & 2430 \\
11- $\beta$-Hydroxyandrosteron & 744 & 45 & 688 & 734 & 754 \\
11- $\beta$-Hydroxyätiocholanolon & 1190 & 77 & 1150 & 1200 & 1170 \\
Pregnandiol & 21200 & 835 & 15600 & 18400 & 20300 \\
Pregnantriol & 3940 & 89 & 2330 & 3420 & 3910 \\
\hline
\end{tabular}

b) Sammelharn von vier $15 \mathrm{j}$ ährigen gesunden Knaben

\begin{tabular}{lrrrr}
\hline & \multicolumn{5}{c}{ Freie Steroide und Glucuronide } \\
& \multicolumn{5}{c}{$\begin{array}{c}\mu \mathrm{g} / 24 \\
\text { S Stdn. }\end{array}$} \\
& $\mathrm{a}$ & $\mathrm{b}$ & $\mathrm{d}$ & $\mathrm{e}$ \\
\hline Androsteron & 654 & $\mathbf{3 4 7}$ & $\mathbf{5 8 8}$ & 641 \\
Åtiocholanolon & 592 & 236 & 557 & 587 \\
Dehydroepiandrosteron & 256 & 87 & 211 & 263 \\
11-Kettoandrosteron & 164 & 34 & 113 & 165 \\
11-Kettoätiocholanolon & 237 & 27 & 189 & 229 \\
11- $\beta$-Hydroxyandrosteron & 86 & 10 & 70 & 87 \\
11- $\beta$-Hydroxyätiocholanolon & 80 & 10 & 71 & 86 \\
Testosteron & 60 & 44 & 55 & 63 \\
Pregnandiol & 283 & 107 & 213 & 290 \\
Pregnantriol & 197 & 84 & 154 & 211 \\
\hline
\end{tabular}


Ausbeuten erhielten wir nach Auftauen im $40^{\circ}$ warmen Wasser, aber auch hierbei waren beachtliche Verluste zu beobachten. Somit begannen wir die Möglichkeit einer besseren Präparation vor dem Einfrieren zu erproben, um die Verluste, die durch den Gefrier- bzw. Auftauvorgang auftreten, auf ein Minimum zu reduzieren. Eine Zugabe von Konservierungsmitteln, wie Toluol oder Thymol, ergab keine eindeutige Verbesserung der Ausbeuten. Es zeigte sich jedoch eine pH-Abhängigkeit der Zersetzung, d. h. bei alkalischen Harnen waren die Verluste im allgemeinen größer als bei den sauren. Allerdings muß gesagt werden, daß die Zersetzung überhaupt von Harn zu Harn sehr verschieden ist. Während wir unter sonst gleichen Bedingungen bei einem Harn starke Zersetzung der Steroide beobachten mußten, fanden wir bei einem anderen, der zur selben Zeit unter gleichen Bedingungen eingefroren und wieder aufgetaut worden war, so gut wie keine Veränderung des Steroidmusters. Im Laufe der Untersuchungen konnten wir folgende Bedingungen als optimal festlegen: Geringe Harnmengen $(100-200 \mathrm{~m} /$ ) erleiden geringere Veränderungen als große (gesamte 24-Stunden-Harnmengen von $1,5-2 \downarrow)$, der günstigste $\mathrm{pH}$-Bereich liegt um 4,0. Ebenfalls günstig macht sich die Zugabe von Chloroform bemerkbar, da dadurch jegliche bakterielle Zersetzung vermieden wird.

Ad 3. Gleichzeitig mit den vorigen Versuchen stellten wir auch Versuche darüber an, wie sich das Steroidmuster eines Harnes bei längerer Aufbewahrung bei $-20^{\circ}$ verändert.

Bei einer Aufbewahrungszeit von 1-2 Monaten stellten wir keine Veränderungen des Hormonmusters fest, die die durch das Auftauen bedingten wesentlich über- schreiten. Bei einem Harn, der 6 Monate bei $-20^{\circ}$ gelagert war, waren die wiedergefundenen Mengen der als Sulfat gebundenen Steroide ziemlich unverändert. Die frei und als Glucuronid ausgeschiedenen Steroide aber zeigen starke Zersetzung.

Durch säulenchromatographische Trennung des Ätherextraktes nach $\beta$-Glucuronidasespaltung erhält man zwei Fraktionen. Fraktion 1 enthält Androsteron, Ätiocholanolon, Dehydroepiandrosteron sowie Testosteron. Fraktion 2 Pregnandiol, Pregnantriol, Pregnantriolon, 11-Ketoandrosteron, 11-Ketoātiocholanolon, 11- $\beta$ - Hydroxyandrosteron, 11- $\beta$-Hydroxyätiocholanolon. In Fraktion 2 fanden wir bei diesem 6 Monate alten Harn annähernd dasselbe Muster wie bei der ersten Analyse, d. h. wir fanden die bekannten Peaks wieder, wenn gleich auch mit veränderter Größe im Verhältnis zur ersten Analyse. Fraktion 1 jedoch (Chromatogramm 1) zeigt keinen bekannten Peak, dafür aber eine Reihe unbekannter Peaks entsprechend den bei der Lagerung gebildeten Artefakten. Daraus kann man schließen, daß sich ein Harn ohne Zugabe von Konservierungsmittel selbst bei $-20^{\circ}$ nur verhältnismäßig kurze Zeit unzersetzt aufbewahren läßt.

Ad 4. Die im vorigen besprochenen Analysenergebnisse untersuchten wir nun noch hinsichtlich der Möglichkeit, aus den Zersetzungsprodukten auf die wirklichen Verhältnisse Rückschlüsse ziehen zu können. Dies ist jedoch nicht möglich. Selbst wenn man die Bedingungen, unter denen die Zersetzung eingetreten ist, genau kennt, ist eine Reproduzierbarkeit der Ergebnisse selbst bei ein und demselben Harn kaum möglich, bei verschiedenen Harnen so gut wie ausgeschlossen.

\section{Literatur}

1. Worrz, H. H. und Sr. J. Clark, Gaschromatography in the analysis of steroid hormones, Plenum Press, New York (1966). 2. HüBENER, H. J. und W. H. STAIB, Biochemie der Nebennierenrindenhormone, Georg Thieme Verlag, Stuttgart (1965). 3. Dorfman, R. I. und F. Ungar, Metabolism of Steroid Hormones, Academic Press, New York (1965). - 4. Currius, H. Ch., Bull. Schweiz. Vereinigung für Klin. Chemie, 12, 30 (1966). -5.
Luukkainen, T., W. J. A. Vanden Heuvel, E. O. A. Hahitit und E. C. Horning, Biochim. biophysica Acta, Amsterdam, 52, 599 (1961). - 6. Horning, E. C., M. G. Horning, N. IKeKawa, E. M. Chambaz, P. I. Jahkonmaki und C. J. W. Brooks, J. Gaschromatography, 5, 283 (1967). - 7. GleispaCH, H., Z. analyt. Chem. 243, 294 (1968). - 8. Bravo, E. L. und R. H. Travis, J. Laborat. Clin. Med., S, Louis, 70, 831 (1967).
Dr. H. Gleispach A 6020 Innsbruck Anichstr. 35 